\title{
Association between XRCC3 Thr241Met polymorphism and risk of osteosarcoma in a Chinese population
}

\author{
J. Guo' ${ }^{1}$, H.C. Lv', R.H. Shi ${ }^{2}$ and W.L. Liu ${ }^{1}$ \\ ${ }^{1}$ The Second Hospital of Inner Mongolia Medical University, Hohhot, China \\ ${ }^{2}$ International Mongolian Medicine Hospital of Inner Mongolia, Hohhot, China \\ Corresponding author: W.L. Liu \\ E-mail: wlliu_liu@126.com
}

Genet. Mol. Res. 14 (4): 16484-16490 (2015)

Received August 15, 2015

Accepted October 28, 2015

Published December 9, 2015

DOI http://dx.doi.org/10.4238/2015.December.9.20

\begin{abstract}
Osteosarcoma is one of the most common bone malignancies in adolescents, and hereditary factors may influence its susceptibility. We assessed the association between XRCC3 Thr241Met polymorphism and susceptibility to osteosarcoma in a Chinese population. Between May 2012 and May 2014, a total of 136 osteosarcoma patients and 136 healthy control subjects were included in our study. The XRCC3 Thr241Met polymorphism was analyzed using a polymerase chain reaction restriction fragment length polymorphism assay. By multiple logistic regression analysis, individuals carrying the Met/Met genotype of XRCC3 Thr241Met were at significantly increased risk of osteosarcoma when compared with the Thr/Thr $(\mathrm{OR}=2.50$, $95 \% \mathrm{Cl}=1.13-5.66)$. The Thr/Met+Met/Met genotype of XRCC3 Thr241Met was furthermore found to be correlated with an elevated increased risk of osteosarcoma when compared with the Thr/Thr genotype (OR $=1.71$, $95 \% \mathrm{Cl}=1.03-2.87$ ), and Met/Met genotype of XRCC3 Thr241Met was associated with an increased risk of osteosarcoma compared to the Thr/ $\operatorname{Thr}(\mathrm{OR}=3.50,95 \% \mathrm{Cl}=1.51-8.79)$. In conclusion, our study firstly reports
\end{abstract}


that XRCC3 Thr241Met gene polymorphism is associated with an elavated risk of osteosarcoma.

Key words: XRCC3 Thr241Met; Polymorphism; Osteosarcoma; Chinese population

\section{INTRODUCTION}

Osteosarcoma is one of the most common bone malignancies in adolescents. Despite the application of multimodal treatment strategies of osteosarcoma (OS), the overall five years survival rate for osteosarcoma is $68 \%$. The development of osteosarcoma is a complex and multifactorial process, involving various environmental and genetic factors (Powers et al., 2010; ). Previous studies have reported that radiation, chemicals exposure and child history of hernias have been suggested as risk factors for this disease (Burningham et al., 2012; Thiagarajan and lyer, 2014). Not all people exposed to similar risk factors develop osteosarcoma, however, which suggests that hereditary factors may influence susceptibility. Several previous studies have reported that gene polymorphisms play an important role in osteosarcoma, including variations in VEGF, FGF2, GSTs, NFKB1, RECQL5 and CTLA-4 genes (He et al., 2014; Zhi et al., 2014; Han et al., 2015; Li et al., 2015; Zhang et al., 2015; Wang et al., 2015).

It is well known that unrepaired DNA damage can cause cell apoptosis and unregulated cell growth, and thus increase the cancer susceptibility. The DNA repair genes are responsible for maintaining cellular functions and homeostasis, and the capacity of DNA repair could be changed by DNSA sequence variations. X-ray repair cross-complementing group 3 (XRCC3) is a member of the DNA repair genes and is involved in the process of homologous recombination repair pathway (Griffin et al., 2000). The function of XRCC3 is to maintain the stability of the genome and repair DNA damage (Griffin et al., 2000). XRCC3 gene is localized on human chromosomes 14q32.3. Codon 241 (Thr241Met) is the most frequent polymorphism in XRCC3, and its polymorphism results in amino acid from $\mathrm{C}$ to $\mathrm{T}$ transition (Shen et al., 1998). Polymorphism in the XRCC3 Thr241Met can influence the function of the encoded protein and thus change the capacity of DNA repair (Matullo et al., 2001). Currently, an association between XRCC3 Thr241Met polymorphism and the risk of osteosarcoma, however, has not been reported. The aim of this study was to the association between XRCC3 Thr241Met polymorphism and susceptibility to osteosarcoma.

\section{MATERIAL AND METHODS}

\section{Study population}

This study was a hospital-based case-control study. Between May 2012 and May 2014, patients with pathologically diagnosed osteosarcoma were enrolled from the Second Hospital of Inner Mongolia Medical University. A total of 136 patients were newly diagnosed and included in our study. A total of 136 control subjects without cancers were selected from the Second Hospital of Inner Mongolia Medical University during the same period. The control subjects were frequently matched to cases by age ( \pm 5 years).

Demographic and clinical information of patients were collected from medical records, including histological type, tumor location and stage. The clinical features of the study subjects are described in Table 1. A signed informed consent form was obtained from every participant (test and 
control) before their inclusion in the study. This protocol was approved by the Second Hospital of Inner Mongolia Medical University.

\section{DNA extraction and SNP genotyping}

Each subject was asked to provide a 5-mL peripheral venous blood for DNA extraction. According to the manufacture's instructions, the TIANamp Blood DNA kit (Tiangen Biotech Co., Ltd., Beijing, China) was taken to extract genomic DNA from peripheral blood samples. The XRCC3 Thr241Met polymorphism was analyzed using a polymerase chain reaction restriction fragment length polymorphism (PCR-RFLP) assay. The forward and reverse primers for the XRCC3 Thr241Met were forward 5'-GGTCGACAGAGTGTCCAAAC-3' and reverse 5'-CTACCCGCAGGAGGGCCACC-3', respectively. The PCR amplification conditions were as follows: a 8 minutes denaturation step at $94^{\circ} \mathrm{C}$ followed by 30 cycles of $95^{\circ} \mathrm{C}$ for 60 seconds (denaturation), $60^{\circ} \mathrm{C}$ for 60 seconds (annealing), and $72^{\circ} \mathrm{C}$ for 1 minute (extension). Amplified products were digested with restriction enzyme of $10 \mathrm{U}$ FastDigest Nlalll at $37^{\circ} \mathrm{C}$ for $10 \mathrm{~min}$, yielding products of $205 \mathrm{bp}$ in length for XRCC3 Thr241Met. The resulting DNA fragments were analyzed by electrophoresis on a $2 \%$ agarose gel stained with ethidium bromide and visualized under UV light. Three patterns were observed: 104, 141 and 170bp band for Met allele, and 141 and 274bp bands for Thr allele.

\section{Statistical analysis}

Statistical differences between osteosarcoma patients and controls regarding demographic and clinical characteristics were assessed by a Chi-squared test or a Fisher's exact test. Departures from the Hardy-Weinberg equilibrium (HWE) were analyzed using a Chi-squared test with one degree of freedom (Genetic Data Analysis program version 1.1). Multiple logistic regression models were established to estimate the association between the XRCC3 Thr241Met polymorphism and osteosarcoma risk. The quantification of statistically significant association was estimated by adjusted odds ratio (OR) and their associated $95 \%$ confidence intervals (Cls). The most common control homozygote of XRCC3 Thr241Met was taken as reference for analysis. Statistical analysis was conducted using SPSS 17.0 (SPSS Inc., Chicago, IL, USA). Differences with two-tailed $P$ values $<0.05$ were considered statistically significant.

\section{RESULTS}

The distributions of demographic and clinical characteristics are shown in Table 1. The mean ages of osteosarcoma patients and controls were $17.82 \pm 5.40$ and $17.31 \pm 5.52$ years, respectively. There were no significant differences in the sexes $\left(X^{2}=0.75, P=0.39\right)$ and ages $\left(X^{2}\right.$ $=0.06, \mathrm{P}=0.80$ ) between osteosarcoma group and the control group. Among the 136 patients, $83(61.03 \%)$ had I-II Enneking stage, 53 (38.97\%) had III Enneking stage, 80 (58.82\%) were osteoblastic type, 21 (15.44\%) were chondroblastic type, 17 (12.50\%) were fibroblastic type, 86 $(63.24 \%)$ were located in the extremities and 50 (36.76\%) were located elsewhere.

Genotype frequencies of the XRCC3 Thr241Met gene in osteosarcoma patients and controls are summaried in Table 2. The observed genotype frequencies of XRCC3 Thr241Met in osteosarcoma patients and controls conformed with the Hardy-Weinberg equilibrium, and the $P$ values of HWE were 0.28 and 0.95 for osteosarcoma patients and controls, respectively. By 
means of a $X^{2}$ test, significant differences were found in the genotype frequencies of XRCC3 Thr241Met between patients and controls in codominant $\left(X^{2}=6.27, P=0.04\right)$, dominant $\left(X^{2}=\right.$ $4.78, P=0.03)$ and recessive models $\left(X^{2}=10.37, P=0.001\right)$. By multiple logistic regression analysis, individuals carrying the Met/Met genotype of XRCC3 Thr241Met were at significantly increased risk of osteosarcoma when compared with the Thr/Thr genotype $(\mathrm{OR}=2.50,95 \% \mathrm{Cl}$ = 1.13-5.66). The Thr/Met+Met/Met genotype of XRCC3 Thr241Met was furthermore found to be correlated with an elevated increased risk of osteosarcoma when compared with the Thr/Thr genotype $(\mathrm{OR}=1.71,95 \% \mathrm{Cl}=1.03-2.87)$, and the Met/Met genotype of XRCC3 Thr241Met was associated with an increased risk of osteosarcoma compared to the Thr/Thr genotype (OR $=3.50,95 \% \mathrm{Cl}=1.51-8.79$ ).

Stratification analysis showed no significant correlation of XRCC3Thr241Met polymorphism with Enneking stage, tumor location, or histological subtype in the risk of osteosarcoma (Table 3). No interaction was moreover identified between XRCC3 Thr241Met polymorphism and genders and ages ( $P$ for interaction $>0.05)$.

Table 1. The demographic and clinical characteristics of patients with osteosarcoma and control subjects.

\begin{tabular}{|c|c|c|c|c|c|c|}
\hline Characteristics & Patients & $\%$ & Controls & $\%$ & $x^{2}$ test & $P$ value \\
\hline \multicolumn{7}{|l|}{ Gender } \\
\hline Female & 52 & 38.24 & 59 & 43.60 & & \\
\hline Male & 84 & 61.76 & 77 & 56.40 & 0.75 & 0.39 \\
\hline \multicolumn{7}{|l|}{ Age, years } \\
\hline$<20$ & 85 & 62.50 & 87 & 63.97 & & \\
\hline$\geq 20$ & 51 & 37.50 & 49 & 36.03 & 0.06 & 0.80 \\
\hline \multicolumn{7}{|l|}{ Enneking stage } \\
\hline$|-| \mid$ & 83 & 61.03 & & & & \\
\hline III & 53 & 38.97 & & & & \\
\hline \multicolumn{7}{|c|}{ Histological subtype } \\
\hline Osteoblastic & 80 & 58.82 & & & & \\
\hline Chondroblastic & 21 & 15.44 & & & & \\
\hline Fibroblastic & 17 & 12.50 & & & & \\
\hline Other & 18 & 13.24 & & & & \\
\hline \multicolumn{7}{|l|}{ Tumor location } \\
\hline Extremities & 86 & 63.24 & & & & \\
\hline Other & 50 & 36.76 & & & & \\
\hline
\end{tabular}

Table 2. Association between XRCC3 Thr241Met gene polymorphism and osteosarcoma risk.

\begin{tabular}{|c|c|c|c|c|c|c|c|c|}
\hline XRCC3 Thr241Met & Patients & $\%$ & Controls & $\%$ & $x^{2}$ value & $P$ value & OR $(95 \% \mathrm{Cl})^{1}$ & $P$ value \\
\hline \multicolumn{9}{|l|}{ Codominant } \\
\hline Thr/Thr & 54 & 39.71 & 70 & 51.47 & & & 1.0 (Ref.) & - \\
\hline Thr/Met & 55 & 40.44 & 52 & 38.24 & & & $1.37(0.79-2.38)$ & 0.23 \\
\hline Met/Met & 27 & 19.85 & 14 & 10.29 & 6.27 & 0.04 & $2.50(1.13-5.66)$ & 0.01 \\
\hline \multicolumn{9}{|l|}{ Dominant } \\
\hline Thr/Thr & 54 & 39.71 & 70 & 51.47 & & & 1.0 (Ref.) & - \\
\hline Thr/Met + Met/Met & 82 & 60.29 & 62 & 45.59 & 4.78 & 0.03 & $1.71(1.03-2.87)$ & 0.03 \\
\hline \multicolumn{9}{|l|}{ Recessive } \\
\hline Thr/Thr + Thr/Met & 109 & 80.15 & 127 & 93.38 & & & 1.0 (Ref.) & - \\
\hline Met/Met & 27 & 19.85 & 9 & 6.62 & 10.37 & 0.001 & $3.50(1.51-8.79)$ & 0.001 \\
\hline
\end{tabular}

${ }^{1}$ Adjusted for sex and age. ORR: odds ratio; $\mathrm{Cl}$ : confidence interval. 
Table 3. Stratification analysis of the association between XRCC3 Thr241Met polymorphism and osteosarcoma risk by clinical characteristics.

\begin{tabular}{|c|c|c|c|c|c|c|c|c|}
\hline Variables & Number & $\%$ & Thr/Thr & $\%$ & Thr/Met + Met/Met & $\%$ & $\mathrm{OR}(95 \% \mathrm{Cl})^{1}$ & $P$ value \\
\hline Enneking stage & & & 54 & & 82 & & & \\
\hline$I-I \mid$ & 83 & 61.03 & 31 & 57.41 & 52 & 63.41 & 1.0 (Ref.) & \\
\hline III & 53 & 38.97 & 23 & 42.59 & 30 & 36.59 & $1.29(0.60-2.75)$ & 0.48 \\
\hline \multicolumn{9}{|l|}{ Tumor location } \\
\hline Other & 50 & 36.76 & 19 & 35.19 & 31 & 37.80 & 1.0 (Ref.) & \\
\hline Extremities & 86 & 63.24 & 35 & 64.81 & 51 & 62.20 & $1.12(0.52-2.45)$ & 0.76 \\
\hline \multicolumn{9}{|l|}{ Tumor metastasis } \\
\hline Osteoblastic & 80 & 58.82 & 31 & 57.41 & 49 & 59.76 & 1.0 (Ref.) & \\
\hline Chondroblastic & 21 & 15.44 & 9 & 16.67 & 12 & 14.63 & $1.19(0.39-3.48)$ & 0.73 \\
\hline Fibroblastic & 17 & 12.5 & 14 & 25.93 & 21 & 25.61 & $1.05(0.43-2.55)$ & 0.9 \\
\hline
\end{tabular}

OR: odds ratio; $\mathrm{Cl}$ : confidence interval.

\section{DISCUSSION}

In this hospital-based case-control study, the role of three important polymorphisms of the XRCC3 Thr241Met gene in osteosarcoma risk as well as their interaction of this polymorphism with clinical and demographic factors in the development of osteosarcoma were assessed. The results of this study revealed that XRCC3 Thr241Met polymorphism is associated with an increased risk of osteosarcoma in codominant, dominant and recessive models, suggesting that variants in $X R C C 3$ Thr241Met are involved in susceptibility to this disease.

It is well known that $X R C C 3$ gene is a member of the Rad51-related protein in human, which plays an important role in homologous recombination to maintain genome integrity and repair DNA damage. Defective double-strand break repair of cells in human could contribute to carcinogenesis. Polymorphisms in XRCC3 result in alteration in DNA repair efficiency, and thus influence the cancer susceptibility in human. Several recent studies have investigated the association between XRCC3 Thr241Met polymorphism and different types of cancers, but the results are contradictory. Some studies have found that XRCC3 Thr241Met polymorphism is associated with a high risk of cancer, such as glioma, hepatocellular carcinoma, colorectal cancer, breast cancer, head and neck cancer and gastric cancer (Duan et al., 2013; Luo et al., 2013; Kayani et al., 2014; Nissar et al., 2014; Qureshi et al., 2014). Duan and colleagues investigated the role of XRCC3 Thr241Met polymorphism in hepatocellular carcinoma, and reported that the Met/Met and Thr/Met+Met/Met genotypes were significantly associated with hepatocellular carcinoma in a Chinese population (Duan et al., 2013). Luo amd colleagues conducted a case-control study with 297 glioma cancer patients and 458 cancer-free controls in a Chinese population, and reported that XRCC3 Thr241Met polymorphism was associated with an elevated risk of gliomas and meningiomas (Luo et al., 2013). Kayani and colleagues investigated the role of XRCC3 Thr241Met gene polymorphism in head and neck cancer and reported that XRCC3 Thr241Met polymorphism was an independent risk factor for head and neck cancer (Kayani et al., 2014). Nissar and colleagues conducted a study with 120 colorectal cancer and 150 healthy subjects in an Indian population, and reported that Thr/Met and Met/Met Genotypes of XRCC3 Thr241Met were corrected with colorectal cancer (Nissar et al., 2014).

However, some studies have shown different results (Xu et al., 2013; Bănescu et al., 2014; Xing et al., 2014; Bashir et al., 2015). Bănescu and colleagues reported that the XRCC3 Thr241Met polymorphism was not associated with the risk of chronic myeloid leukemia (Bănescu et al., 2014). $\mathrm{Xu}$ and colleagues revealed that XRCC3 Thr241Met polymorphism was not associated with the development of lung cancer in a meta-analysis (Xu et al., 2014). Bashir and colleagues conducted 
a study with 80 gastric cancer patients and 70 healthy controls, and reported that the Thr/Met and Met/Met genotypes of XRCC3 Thr241Met may not play a role in the development of gastric cancer (Bashir et al., 2015). These discrepancies between different studies may result from differences in the cancer types, and study designs between studies as well as sample sizes.

To date, no association between XRCC3 Thr241Met and risk of osteosarcoma has been reported. Our study firstly reports that XRCC 3 Thr241Met gene polymorphism is associated with an elevated risk of osteosarcoma. These findings certainly warrant further investigation using larger sample sizes for confirmation.

\section{Conflicts of interest}

The authors declare no conflict of interest.

\section{ACKNOWLEDGMENTS}

We thank for the staffs in the Second Hospital of Inner Mongolia Medical University for collection of the blood samples.

\section{REFERENCES}

Bashir H, Majid S, Hamid R, Farooq R, et al. (2015). Polymorphism of the XRCC3 gene and risk of gastric cancer in a Kashmiri population: a case-control study. Eur. J. Cancer Prev. 24: 167-175.

Bănescu C, Trifa AP, Demian S, Benedek Lazar E, et al (2014). Polymorphism of XRCC1, XRCC3, and XPD genes and risk of chronic myeloid leukemia. Biomed. Res. Int. 2014: 213790.

Bovee JV and Hogendoorn PC (2010). Molecular pathology of sarcomas: Concepts and clinical implications. Virchows Archiv. 456: 193-199.

Burningham Z, Hashibe M, Spector L, Schiffman JD (2012). The epidemiology of sarcoma. Clin. Sarcoma Res. 2: 14.

Duan C, Zhang W, Lu J, Wu H, et al. (2013). DNA repair gene XRCC3 Thr241Met polymorphism and hepatocellular carcinoma risk. Tumour Biol. 34: 2827-2834.

Griffin CS, Simpson PJ, Wilson CR and Thacker J (2000). Mammalian recombination-repair genes xrcc2 and xrcc3 promote correct chromosome segregation. Nat. Cell Biol. 2:757-761.

Han J, Deng W, Wang L and Qi W (2015). Null genotypes of glutathione S-transferase $\mu 1$ and glutathione S-transferase $\theta 1$ are associated with osteosarcoma risk: A meta-analysis. Oncol. Lett. 9: 1912-1916.

He J, Wang J, Wang D, Dai S, et al. (2014). Association between CTLA-4 genetic polymorphisms and susceptibility to osteosarcoma in Chinese Han population. Endocrine. 45: 325-330.

Kayani MA, Khan S, Baig RM, Mahjabeen I (2014). Association of RAD 51135 G/C, 172 G/T and XRCC3 Thr241Met gene polymorphisms with increased risk of head and neck cancer. Asian Pac. J. Cancer Prev. 15: 10457-10462.

Li X, Zhang C, Qiao W, Zhou X, et al. (2015). NFKB1 -94ins/del ATTG polymorphism increases osteosarcoma risk in a Chinese Han population. Int. J. Clin. Exp. Med. 8: 1420-1423.

Luo KQ, Mu SQ, Wu ZX, Shi YN, et al. (2013). Polymorphisms in DNA repair genes and risk of glioma and meningioma. Asian Pac. J. Cancer Prev. 14: 449-452.

Matullo G, Guarrera S, Carturan S, Peluso M, et al. (2001). DNA repair gene polymorphisms, bulky DNA adducts in white blood cells and bladder cancer in a case-control study. Int. J. Cancer. 92: 562-567.

Nissar S, Sameer AS, Lone TA, Chowdri NA, et al. (2014). XRCC3 Thr241Met gene polymorphism and risk of colorectal cancer in Kashmir: a case control study. Asian Pac. J. Cancer Prev. 15: 9621-9625.

Powers M, Zhang W, Lopez-Terrada D, Czerniak BA, et al. (2010). The molecular pathology of sarcomas. Cancer biomarkers: section A of Disease markers. 9: 475-491.

Qureshi Z, Mahjabeen I, Baig R and Kayani M (2014). Correlation between selected XRCC2, XRCC3 and RAD51 gene polymorphisms and primary breast cancer in women in Pakistan. Asian Pac. J. Cancer Prev. 15: 10225-10229.

Shen MR, Jones IM and Mohrenweiser H (1998). Nonconservative amino acid substitution variants exist at polymorphic frequency in DNA repair genes in healthy humans. Cancer Res. 58: 604-608. 
Thiagarajan A and lyer NG (2014). Radiation-induced sarcomas of the head and neck. World J. Clin. Oncol. 5: 973-981.

Wang J, Liu H, Liu X, Qi X (2015). Effect of variation of FGF2 genotypes on the risk of osteosarcoma susceptibly: a case control study. Int. J. Clin. Exp. Med. 8: 6114-6118.

Xing ZS, Zhu G, Yang YL, Feng GQ, et al. (2014). Meta analysis of XRCC3 Thr241Met polymorphism and lung cancer susceptibility of populations in East Asia. Asian Pac. J. Trop. Med. 7: 483-487.

Xu YH, Gu LP, Sun YJ, Cheng BJ, et al. (2013). No significant association between the XRCC3 Thr241Met polymorphism and lung cancer risk: a meta-analysis. Tumour Biol. 34: 865-874.

Zhang G, Bai R, Zhang T, Zhang H, et al. (2015). Investigation of the role of VEGF gene polymorphisms in the risk of osteosarcoma. Genet. Mol. Res. 14: 8283-8289.

Zhi LQ, Ma W, Zhang H, Zeng SX, et al. (2014). Association of RECQL5 gene polymorphisms and osteosarcoma in a Chinese Han population. Tumour Biol. 35: 3255-3259. 